\title{
Experimental Study on Methane Yield influenced by Biogas Project Fermentation Temperature
}

\author{
Man Zhou ${ }^{1}$,Luyao Guo ${ }^{1}$,Siqi Liu ${ }^{1}$,Zhiyong Zou ${ }^{2, *}$ \\ 1. College of Water Resources and Hydropower, Sichuan Agricultural University, Ya'an, Sichuan Province, China \\ 2. College of Mechanical and Electronic Engineering, Sichuan Agricultural University, Ya'an, Sichuan Province, China \\ *Corresponding author: Zhiyong Zou, zouziyong111@163.com
}

\begin{abstract}
We tried to understand the differences in the biogas production characteristics of anaerobic sludge digestion at moderate and high temperature. Based on the three-stage theory of anaerobic fermentation, using cellulose, glucose and acetate as substrates, this study compared and analyzed the methanogenic characteristics of anaerobic sludge fermentation at moderate and high temperature. The test results show that the special methane activity (SMA) of acetate, glucose and cellulose at $35^{\circ} \mathrm{C}$ was $66.98 \mathrm{~mL} \cdot \mathrm{gVS}^{-1} \mathrm{~d}^{-1}, \quad 36.11 \mathrm{~mL} \cdot \mathrm{gVS}^{-1} \mathrm{~d}^{-1}$ and $23.33 \mathrm{~mL} \cdot \mathrm{gVS}^{-1} \mathrm{~d}$ 1 ,respectively, and that at $55{ }^{\circ} \mathrm{C}$ was $69.05 \mathrm{~mL} \cdot \mathrm{gVS}^{-1} \cdot \mathrm{d}^{-}$

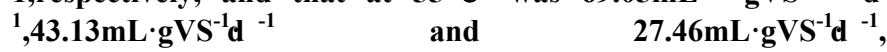
respectively.Compared with moderate temperature anaerobic sludge fermentation, the high temperature anaerobic sludge fermentation has a higher activity, and is featured by shorter hydrolysis time, faster digestion rate and higher methane yield,but the methane concentration of the biogas produced by high temperature anaerobic sludge fermentation is lower than that of the moderate temperature sludge fermentation. Meanwhile, the net energy balance under different temperature conditions was analyzed. Since the high-temperature anaerobic fermentation requires more energy, it is economically disadvantageous. In addition, high-temperature anaerobic fermentation can easily produce ammonia nitrogen inhibition, so the advantage of intermediate temperature fermentation is more obvious.
\end{abstract}

Keywords-Anaerobic; Fermentation; Temperature; High temperature; Sludge activity; Methane

\section{INTRODUCTION}

With the improvement of people's living conditions, the proportion of kitchen garbage in the domestic garbage has become higher and higher; and the discharge of kitchen garbage has naturally become a hot issue in environmental protection [1]. The organic substances in the kitchen garbage mainly include starch, cellulose, protein and fat. In recent years, the demand for renewable energy and the severe environmental problems currently faced by us have made the anaerobic digestion and utilization of biomass waste such as restaurant and kitchen garbage more and more concerned [2 3]. Biogas fermentation is a biochemical process carried out by microorganisms under anaerobic conditions and mainly involves different groups of obligate anaerobic microorganisms (strictly anaerobic) and facultative anaerobic microorganisms (having aerobic microorganisms' functions under aerobic conditions). The biochemical process of microorganisms converts organic raw materials into biogas that mainly includes methane $(50 \% \sim 70 \%)$ and carbon dioxide $(30 \% \sim 50 \%)$, as well as a small amount of hydrogen sulfide. Most of the fermentation raw material is converted into biogas products by microorganisms, and only a few are used for the growth and reproduction of microorganisms. The advantage of anaerobic digestion of kitchen garbage is using microorganisms to degrade organic components, which not only has higher waste treatment efficiency but also can obtain organic fertilizer and biogas energy. In this process, the quantity and quality of the inoculum are very important for the performance and stability of the methanogenic stage in the anaerobic digestion [4].

Temperature is an important factor affecting the life activities of microorganisms, and its influence over the anaerobic microorganisms and biogas fermentation process is particularly obvious [5]. Various kinds of microorganisms grow in certain temperature ranges. According to the temperature ranges in which microorganisms grow, microorganisms are conventionally classified into three types. A. Psychrophilic microorganisms, growth temperature $5 \sim 20^{\circ} \mathrm{C}$. B. Mesophilic microorganisms, growth temperature $20 \sim 42{ }^{\circ} \mathrm{C}$. C. Thermophilic microorganisms, growth temperature $42 \sim 75^{\circ} \mathrm{C}$. Correspondingly, biogas fermentation is also divided into normal temperature, moderate temperature $\left(35^{\circ} \mathrm{C}\right)$ and high temperature $\left(55^{\circ} \mathrm{C}\right)$. The higher the temperature is, the faster the fermentation speed is, and the energy consumption for heating is also higher. On the contrary, the lower the temperature is, the slower the fermentation speed is. Meanwhile, under different temperature conditions, the intermediate products and the final products are different due to the differences in the microbial structures and metabolic pathways. There are researchers who used two-phase anaerobic digestion to treat kitchen garbage [5], their research showed that at 4 temperatures $\left(25^{\circ} \mathrm{C}, 37^{\circ} \mathrm{C}, 40^{\circ} \mathrm{C}\right.$ and $\left.50^{\circ} \mathrm{C}\right)$, the maximum volatile fatty acids (VFAs) percentage was obtained at $37^{\circ} \mathrm{C}$. It mainly included formic acid and acetic acid as well as a small amount of propionic acid and butyric acid, and the percentage of methane at $50^{\circ} \mathrm{C}$ is also lower than that at $37^{\circ} \mathrm{C}$. The SMA is an important physiological activity indicator of methanogens, it refers to the maximum methane yield formed by the conversion of soluble substrate per unit of sludge per unit time, its common unit is milliliter of methane produced by one gram of volatile solid sludge in one day, $\mathrm{mLCH}_{4} \cdot \mathrm{gVS}^{-1} \mathrm{~d}^{-1}$, since the COD removed from the feed liquids are mainly converted to $\mathrm{CH}_{4}$, the specific methanogenic activity indicates the potential methanogenic capacity of the sludge.

In order to study the gas production characteristics of moderate temperature and high temperature sludge fermentation, the differences in the methanogenic activity 
characteristics of the kitchen garbage sludge at moderate and high temperature were investigated. Based on the three-stage theory of anaerobic fermentation; using cellulose, glucose and acetic acid as substrates, this study conducted the specific methanogenic activity test for the two kinds of sludge to try to clarify the gas production mechanism of moderate temperature and high temperature sludge fermentation; and provide basis for the screening of inoculated sludge.

\section{MATERIALS AND METHODS}

\section{A. Characteristics of Raw Materials}

The inoculum sludge was obtained from a laboratoryoperated moderate and high temperature kitchen garbage anaerobic digestion reactor, it was stored at room temperature for 2 months as starvation treatment and the large particulate matter was removed using $2 \mathrm{~mm}$ sieve. See Table 1 for sludge's characteristics. See Table 2 for the typical trace element concentration of the sludge.

TABLE I. Characteristics of ANAERobic Digestion Sludge

\begin{tabular}{ccccc}
\hline Item & TS /\%,w.b. & VS /\%,w.b. & C/N,d.b. & pH \\
\hline $\begin{array}{c}\text { Mesophilic } \\
\text { sludge }\end{array}$ & 6.32 & 3.44 & 36.92 & 7.41 \\
$\begin{array}{c}\text { Thermophili } \\
\text { c sludge }\end{array}$ & 26.91 & 25.23 & 22.01 & 4.51 \\
\hline
\end{tabular}

Note: w.b., web base; d.b., dry base.

TABLE II. TyPICAL TRACE ELEMENT CONCENTRATION OF ANAEROBIC DIGESTION SLUDGE

\begin{tabular}{cccccccc}
\hline Item & B & Cd & Cr & Co & Cu & Fe & Mn \\
\hline $\begin{array}{c}\text { Mesophilic } \\
\text { sludge }\end{array}$ & 24.5 & 2.8 & 131.5 & 18.7 & 46.3 & 3652 & 465.8 \\
$\begin{array}{c}\text { Thermophilic } \\
\text { sludge }\end{array}$ & 28.2 & 1.4 & 154.2 & 16.3 & 2.9 & 3249 & 310.6 \\
\hline
\end{tabular}

\section{B. Test Device}

The experimental devices are shown in Fig.1. A $500 \mathrm{~mL}$ reagent bottle was used as the anaerobic digestion bottle (effective volume $400 \mathrm{~mL}$ ) and another $500 \mathrm{~mL}$ reagent bottle was used as the water collection device. The reaction was performed in a $35^{\circ} \mathrm{C}$ constant temperature water bath, and the gas yield was recorded on a daily basis (the discharged lye volume represents the volume of produced methane) until $80 \%$ of the substrate VFAs has been used.

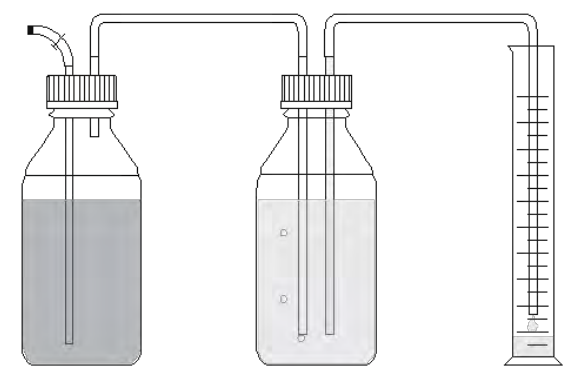

Fig. 1. Anaerobic Fermentation Test Device

\section{Test Method}

Using cellulose, glucose and sodium acetate as substrates; the amount of added COD was converted to $2 \mathrm{~g} \mathrm{COD}$, add $\mathrm{CaO}$ to adjust the $\mathrm{pH}$ to 7.0 .

The calculation method is as follows:

$$
S M A=\frac{24 R}{F \cdot V \cdot V S}
$$

In which: SMA - Specific Methanogenic Activity;

$\mathrm{R}$ - Methanogenic speed (i.e. average slope of the maximum active interval in the curve), $\mathrm{ml} \mathrm{CH}_{4}{ }^{-1}$; the maximum activity interval should cover at least $50 \%$ of the utilized substrate VFAs.

F - Conversion coefficient of saturated water vaporincluded methane volume and COD in grams (Table 1);

$\mathrm{V}$ - Volume of liquid in the reactor, L;

VS - The sludge concentration in the reactor, $\mathrm{gVS} \cdot \mathrm{L}^{-1}$.

\section{Analysis Method}

TS and VS were determined by gravimetric method; gas volumetric measurement was performed by saturated sodium carbonate solution displacement method; $\mathrm{pH}$ was determined by portable $\mathrm{pH}$ meter; and elements were determined by atomic absorption spectrophotometer.

\section{RESUlTS AND DISCUSSION}

\section{A. Biochemical Process of Anaerobic Fermentation}

Anaerobic fermentation utilizes the metabolic effects of facultative anaerobic and obligate anaerobic microorganisms to convert complex organic matter into biogas, water, and small amounts of cellular material under anaerobic conditions. The biogas fermentation of organic matter mainly includes three stages: liquefaction stage, hydrogen production and acetogenesis stage as well as methanogenic stage [6].

Using cellulose, glucose and sodium acetate as the typical substrates for above three stages to investigate the degradation process, it can be seen that the anaerobic fermentation of high temperature and moderate temperature sludge fermentation is essentially the same, in which methane is the final product, but the fermentation process is a complex process in which various microorganisms participate. This process involves a wide variety of microorganisms and huge amount of data. Biogas fermentation microorganisms of various taxa convert various substances according to their nutritional requirements. The process conditions of biogas fermentation and their control are used to provide the optimum conditions for various microorganisms [7]. Under different temperature conditions, there certainly have differences in the bacteria floras and metabolic pathways involved in the two different fermentation systems. Three stages of anaerobic fermentation: The first stage, hydrolysis and fermentation. During this phase, complex organic matters are hydrolyzed and fermented by microorganisms (fermentation bacteria). The polysaccharides are first hydrolyzed to monosaccharides and then further fermented into ethanol and fatty acids through the glycolysis 
pathway. Proteins are first hydrolyzed to amino acids and then deaminated to produce fatty acids and ammonia. Lipids are converted to fatty acids and glycerin and then converted to fatty acids and alcohols. The second stage: Hydrogen production, acetogenesis production (i.e. acidification stage). Under the effect of hydrogen-producing and acetogens, the intermediate products such as fatty acids (propionic acid, butyric acid) and alcohols (ethanol) and other water soluble small molecules produced in the first stage, except for formic acid, acetic acid, methylamine and methanol, are converted to acetic acid, $\mathrm{H}_{2}$ and $\mathrm{CO}_{2}$. The third stage: The methanogenic stage. Methanogens convert substrates such as formic acid, acetic acid, methylamine, methanol and $\left(\mathrm{H}_{2}+\mathrm{CO}_{2}\right)$ into methane through different pathways; the most important substrates are acetic acid and $\left(\mathrm{H}_{2}+\mathrm{CO}_{2}\right)$. About $70 \%$ of methane in the anaerobic digestion comes from the decomposition of acetic acid, and a small amount comes from the synthesis of $\mathrm{H}_{2}$ and $\mathrm{CO}_{2}$. Therefore, from the viewpoint of the change in the physical properties of the fermentation raw materials, as a result of the hydrolysis, the suspended solid organic matters are dissolved, which is called "liquefaction".

\section{B. Analysis of Specific Methanogenic Activity (SMA)}

Methanogens are the core of biogas fermentation microorganisms; the activity of methanogens is an important parameter reflecting the performance of anaerobic sludge; whether the anaerobic sludge with high activity can be formed and maintained is the key to the efficient operation of modern anaerobic fermentation reactors.

From the perspective of digestion time (Fig.2 and Fig. 3), among the three substrates, the digestion time of acetic acid in two kinds of sludge was the same, the digestion time of other two substrates in the high temperature sludge, glucose and starch, was shorter, the difference in the digestion time of cellulose was biggest. This is mainly due to the degradation reaction process; cellulose needs to be hydrolyzed to glucose, followed by glucose acidification to produce acetic acid. Acetic acid is the substrate that can be directly used by methanogens. Therefore, the speed of acetic acid fermentation is the fastest, followed by glucose, and cellulose is the slowest. High temperature sludge is more effective in degrading cellulose and may contain a more abundant hydrolytic bacteria flora.

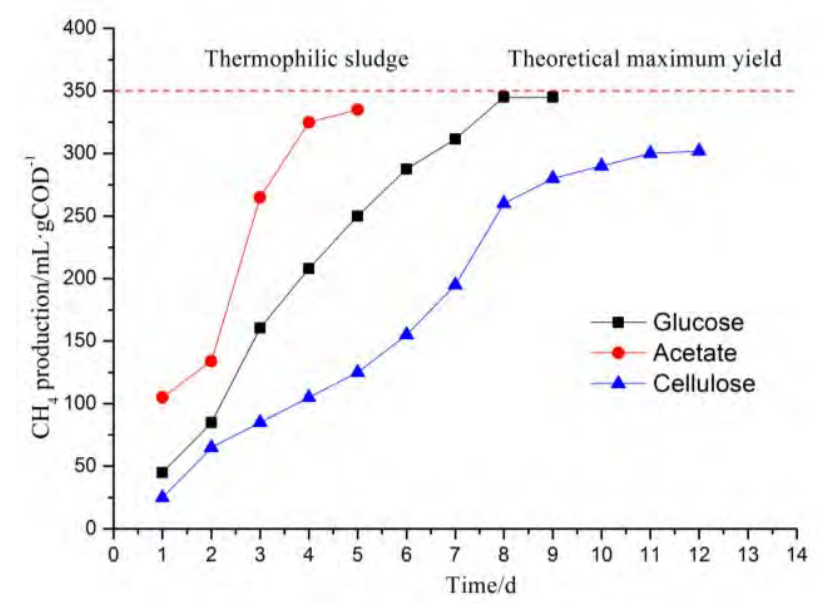

Fig. 2. Cumulative methanogenic curve of high temperature sludge
From the perspective of gas yield (Fig.2, Fig.3 and Fig.4), for both kinds of sludge, the gas volume produced by acetic acid was highest, followed by glucose, and cellulose was the lowest. The gas yields of the two kinds of sludge taking acetic acid and glucose as substrates were similar, while the two kinds of sludge taking cellulose as the substrate were quite different. Compared to substrate anaerobic fermentation sludge, high temperature anaerobic sludge digestion of cellulose had a higher methane yield. This may be due to two reasons: On the one hand, some of the products in the fermentation process are not completely degraded; on the other hand, the degradation products have not been completely converted to methane and are stored as living substances in the cells for metabolic utilization.

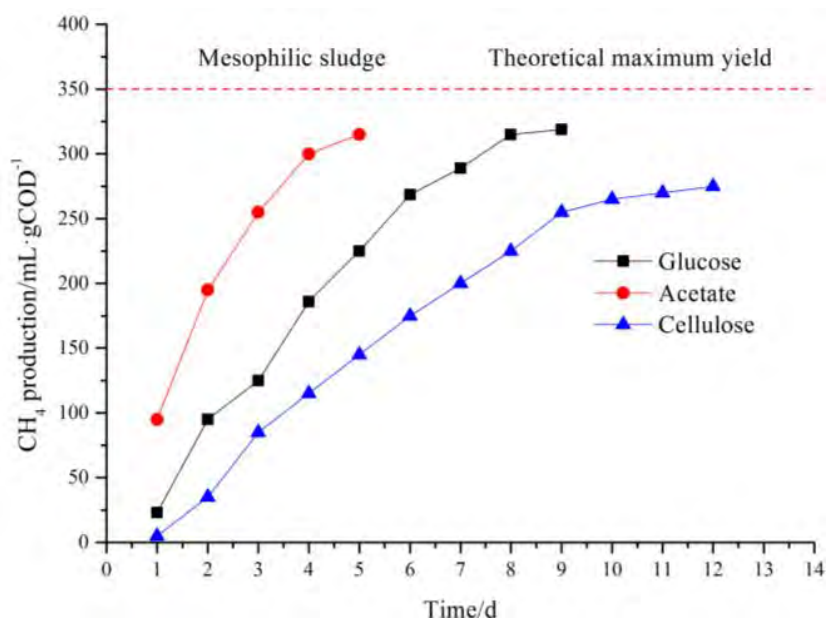

Fig. 3. Cumulative methanogenic curve of moderate temperature sludge fermentation

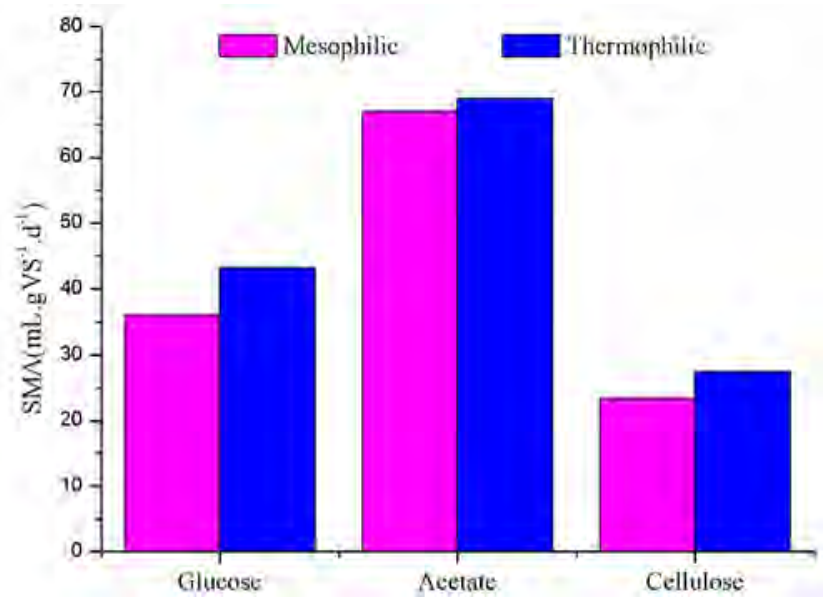

Fig. 4. Comparison of the specific methanogenic activity of different substrates

From the perspective of biogas concentration, the average methane concentration of high temperature sludge was $60.5 \%$; while the average methane concentration of moderate temperature anaerobic fermentation sludge was $63.0 \%$, the average methane content of high temperature sludge was slightly higher than that of moderate temperature sludge, the potential reason is that under the high temperature conditions, the microbial flora structure and metabolic pathways change, 
the activity of methanogens increases and the metabolism is faster, more energy is needed for self-consumption, so the methane yield is relatively reduced. It has been reported in the literature that different types of microorganisms require different temperatures during the biogas fermentation, there have various microorganisms in the reactor. For example, only the thermophilic microorganisms can grow in high temperature biogas fermentation reactor. In each temperature range, as the temperature increases, the microbial growth speed gradually increases and reaches a maximum value, which is the optimum growth temperature for microorganisms; the growth speed of the microorganism rapidly declines after exceeding the optimum temperature.

\section{The Optimum Fermentation Temperature Based on Net Energy}

So far, most biogas fermentation systems are operating in the moderate temperature range, within this range, the speed of the biogas fermentation increases by about 1 times for every $10^{\circ} \mathrm{C}$ increase in temperature; after the temperature is lower than the optimum temperature, the speed of biogas fermentation decreases by $11 \%$ for $1{ }^{\circ} \mathrm{C}$ decrease. At present, the most common temperature for medium temperature fermentation is $30 \sim 40^{\circ} \mathrm{C}$, and its optimum fermentation temperature is $35 \sim 40^{\circ} \mathrm{C}$. The high temperature fermentation process normally runs at $50-60^{\circ} \mathrm{C}$. The reaction speed of high temperature fermentation is $25 \% \sim 50 \%$ higher than that of moderate temperature fermentation, its biogas yield is also higher, but the percentage of methane in the biogas is lower than that of the moderate temperature, and it is susceptible to the changes in operating conditions and the environment, which easily causes the accumulation of volatile acids. However, for the treatment of feed liquids containing pathogenic bacteria and parasitic egg, high temperature fermentation can achieve the desired hygienic effect. The high temperature digestion requires more energy; it is usually not suitable for treatment production in enormous quantities. The normal temperature biogas fermentation process has a lower reactor load because its sludge activity is significantly lower than that of moderate and high temperatures. For some low temperature wastewater, it will consume too much energy to increase the fermentation temperature of the wastewater. In the absence of economic heating energy, the normal temperature fermentation process is also an alternative solution.

However, the net energy obtained is directly related to the feed VS concentration because the absolute methane potential depends on the amount of VS. It has been reported in the literature [5] that if the concentration of input VS is higher than $45000 \mathrm{mg} \cdot \mathrm{L}^{-1}, 55^{\circ} \mathrm{C}$ is more economical than $35^{\circ} \mathrm{C}$ because the methane yield at $55^{\circ} \mathrm{C}$ overcomes the consumption of heat supply. However, if the concentration of input VS is lower than $45000 \mathrm{mg} \cdot \mathrm{L}^{-1}, 35^{\circ} \mathrm{C}$ is more favorable than $55^{\circ} \mathrm{C}$. In addition, temperature has a strong influence over the free ammonia concentration; free ammonia is a stronger inhibitor than ammonia nitrogen. The concentration of free ammonia increases with the increase in temperature, therefore, it is necessary to carefully consider increasing the methane yield by increasing the temperature, because the ammonia inhibition will be increased as well. The specific used fermentation temperature depends on the factors such as raw material temperature, local air temperature, heating personnel, net energy output and other comprehensive factors.

In the most suitable range, the small fluctuations in temperature (for example, $1 \sim 3^{\circ} \mathrm{C}$ ) will not have a significant effect on the biogas fermentation process. If the temperature drop is too large, the activity of the sludge will be significantly reduced, and the corresponding reactor load should also be properly reduced to prevent the problems such as acid accumulation in the reactor due to excessive load.

\section{CONCLUSIONS}

The activity of methanogens is an important parameter reflecting the performance of anaerobic sludge; whether the anaerobic sludge with high activity can be formed and maintained is the key to the efficient operation of modern anaerobic fermentation reactors. Based on the three-stage theory of anaerobic fermentation; using cellulose, glucose and acetic acid as substrates, this study compared and analyzed the methanogenic characteristics of anaerobic sludge fermentation at moderate and high temperature. The test results show that the high temperature fermentation is more conducive to hydrolysis, and has a faster digestion speed and higher methane yield, but its methane concentration in the biogas is lower than that of the moderate temperature sludge fermentation. Meanwhile, the net energy balance under different temperature conditions was analyzed, because the high temperature fermentation requires more energy, it is economically disadvantageous, and high temperature fermentation can easily cause ammonia nitrogen inhibition, so the advantage of moderate temperature fermentation is more obvious.

\section{ACKNOWLEDGEMENTS}

The research work was supported Key Projects in Sichuan Province Department of Education No. 15ZB0008.

Zhiyong Zou* (Corresponding author) is a Lecturer in Sichuan Agriculture University. (Em: zouziyong111@163.com)

\section{REFERENCES}

[1] C. Liu, H. Li, Y. Zhang, "Characterization of methanogenic activity during high-solids anaerobic digestion of sewage sludge," Biochemical Engineering Journal, issue 109, pp. 96-100, 2016.

[2] N.I. Muhammad, G.T.I. Mohd, R. Omar, "Production of biogas from solid organic wastes through anaerobic digestion: a review," Appl Microbiol Biotechnol, vol.95, issue2, pp. 321-329, 2012.

[3] E. Iacovidou, D.G. Ohandja, N. Voulvoulis, "Food waste co-digestion with sewage sludge--realising its potential in the UK," Journal of Environmental Management, vol112, issue 24, pp. 267-274, 2012.

[4] D. Vrieze, J. Saunders, W. Boon, "Ammonia and emperature determine potential clustering in the anaerobic digestion microbiome," Water Research, issue75, pp. 312-323, 2015.

[5] C.L. Mao, Y.Z. Feng, X.J. Wang, G.X. Ren, "Review on research achievements of biogas from anaerobic digestion," Renewable Sustainable Energy Review, issue 45, pp. 540-555, 2015.

[6] Q. Lin, V.J. De, C. Li, “Temperature regulates deterministic processes and the succession of microbial interactions in anaerobic digestion process," Water Research, issue 123, pp. 134-143 2017.

[7] J. Mata-Alvarez, P. Llabres, S. Mace, "Anaerobic digestion of organic solid wastes an overview of research achievements and perspectives," Bioresource Technology, vol.74, issue1, pp. 3-16, 2000. 